\title{
Mapping of Coulomb Gases and Sine-Gordon Models to Statistics of Random Surfaces
}

\section{Citation}

Imambekov, Adilet, Vladimir Gritsev, and Eugene Demler. 2008. Mapping of Coulomb gases and sine-Gordon models to statistics of random surfaces. Physical Review A 77: 063606.

\section{Published Version}

doi://10.1103/PhysRevA.77.063606

\section{Permanent link}

http://nrs.harvard.edu/urn-3:HUL.InstRepos:13421140

\section{Terms of Use}

This article was downloaded from Harvard University's DASH repository, and is made available under the terms and conditions applicable to Open Access Policy Articles, as set forth at http:// nrs.harvard.edu/urn-3:HUL.InstRepos:dash.current.terms-of-use\#OAP

\section{Share Your Story}

The Harvard community has made this article openly available.

Please share how this access benefits you. Submit a story.

Accessibility 


\title{
Mapping of Coulomb gases and sine-Gordon models to statistics of random surfaces
}

\author{
Adilet Imambekov, ${ }^{1,2}$ Vladimir Gritsev, ${ }^{1}$ and Eugene Demler ${ }^{1}$ \\ ${ }^{1}$ Department of Physics, Harvard University, Cambridge, Massachusetts 02138, USA \\ ${ }^{2}$ Department of Physics, Yale University, New Haven, Connecticut 06520, USA
}

(Dated: June 13, 2008)

\begin{abstract}
We introduce a new class of sine-Gordon models, for which the interaction term is present in a region different from the domain over which the quadratic part is defined. We develop a nonperturbative approach for calculating partition functions of such models, which relies on mapping them to statistical properties of random surfaces. As a specific application of our method, we consider the problem of calculating the amplitude of interference fringes in experiments with two independent low dimensional Bose gases. We calculate full distribution functions of interference amplitude for one-dimensional and two-dimensional gases with nonzero temperatures.
\end{abstract}

\section{INTRODUCTION}

Sine-Gordon (SG) models and their Coulomb gas representations appear as a low energy effective theory for many types of physical systems. The so-called "bulk" SG model describes the Berezinskii-Kosterlitz-Thouless (BKT) transition in two-dimensional superfluids [1, 2, 3] and the superfluid to insulator transition of Cooper pairs in a chain of Josephson junctions [4]. The socalled "boundary" SG model [5] can be used to describe the Chakravarty-Schmid transition in a single Josephson junction with dissipation [6] and a quantum impurity problem [7] in one dimension (1D). Powerful theoretical techniques have been developed for studying such SG models including Bethe ansatz solutions [8], renormalization group analysis (see e.g., Ref. [3]), and functional renormalization group [9]. Recent theoretical work suggested that there is another class of SG models which is important for several kinds of physical systems. Such models can be described by the action

$$
S(g)=\pi K \int_{\Omega}(\vec{\nabla} \phi)^{2} d x d \tau+2 \int_{\omega} g \cos (2 \pi \phi) d x d \tau,
$$

where the interaction term $\cos (2 \pi \phi)$ is present in the spatial region $\omega$ (or space-time region for quantum problems), which is only a part of the domain $\Omega$ over which the noninteracting part $(\vec{\nabla} \phi)^{2}$ is defined. This should be contrasted to the bulk SG models, in which the interaction term is present in the entire region $\Omega$, and to the boundary SG models, in which the interaction term is present on a line. We note that models with inhomogeneous $g(x, \tau)$ can be considered using methods developed in the paper as well, but for concreteness we will consider only constant $g$. Model (10) interpolates between the bulk and boundary SG models, and we will refer to it as the interior sine-Gordon (ISG) model. Here are a few examples of physical systems that can be described by such models. The first example is the problem of "interwire coherence" 10] in which wires are brought together over a finite length $l$ and separated on both ends. The cos-term describes the correlated umklapp electron scattering in the two wires $\left(+2 k_{F}\right.$ scattering in one wire accompanied by $-2 k_{F}$ scattering in the other wire). The quantum space-time action of this system has the form of Eq. (1) with $\Omega=[-\infty, \infty]_{x} \times[0, \beta]_{\tau}$ and $\omega=[-l / 2, l / 2]_{x} \times[0, \beta]_{\tau}$. Another example comes from a system of quantum particles in one dimension (e.g., electrons or Cooper pairs in a wire, or ultracold atoms in a weak optical trap) with a periodic potential present in a finite region of the system. The cos-term comes from the umklapp scattering on the external potential and is limited to the finite region in the interior of the system. The third example is the problem of distribution functions of interference fringe amplitudes (DFIFA) for a pair of independent low-dimensional condensates [11, 12, 13]. Individual moments of the distribution function can be represented as a microcanonical partition function of Coulomb gases [14, 15, 16], with positions of Coulomb charges restricted to the part of the system from which interference patterns have been extracted. The latter is typically smaller than the total system size. For example, in the case of large 2D condensates we have $\Omega=[-\infty, \infty]_{x} \times[-\infty, \infty]_{\tau}$, and when the interference pattern is extracted from the area $l \times l$, we have $\omega=[-l / 2, l / 2]_{x} \times[-l / 2, l / 2]_{\tau}$. The relation between the DFIFA and the partition function of the ISG model will be outlined below and has also been discussed in Refs. [14, 15, 16].

In this paper we develop a nonperturbative approach to calculating partition functions of a wide class of SG models and Coulomb gases, which relies on the mapping of their partition functions to certain problems of statistics of random surfaces. We point out that our method does not rely on the existence of the exact solutions of SG models, but uses the structure of the multi-point correlation functions in the absence of interactions. We also note that a suitable extension of our method can be used to compute correlation functions of SG models in equilibrium and non-equilibrium situations. The particular strength of our approach is that it can be applied to study ISG models, which cannot be analyzed by other theoretical methods. As a concrete application of our method we calculate DFIFA for both 1D and 2D condensates. We point out that earlier theoretical work on interference experiments focused on 1D systems with periodic boundary conditions (PBC) [15]. While these boundary conditions are extremely artificial from the point of view of realis- 
tic experiments, they allow one to relate the DFIFA to the quantum impurity problem [7] and use certain exact results about the latter [17]. Methods used in Ref. [15] can not be generalized either to the realistic case of open boundary conditions (OBC) (i.e., interference patterns extracted from the interior of a large system) or 2D systems, but these cases can be analyzed using the method discussed in this paper. We emphasize, however, that the main goal of this paper is to introduce an approach to the analysis of SG models and the problem of DFIFA is just one example that illustrates the power of the new method.

\section{MAPPING}

The partition function corresponding to the action (1) is given by $Z(g)=\int \mathcal{D} \phi e^{-S(g)} / \int \mathcal{D} \phi e^{-S(0)}$. By expanding $Z(g)$ in powers of $g$ we arrive at the grand canonical partition function of the Coulomb gas [18]

$$
\left.Z(g)=\sum_{n=0}^{n=\infty} \frac{g^{2 n}}{(n !)^{2}} Z_{2 n}, \text { where } Z_{2 n}=\int_{\omega} \ldots \int_{\omega} d^{2} \vec{u}_{1} \ldots d^{2} \vec{v}_{n} e^{\frac{1}{K}\left(\sum_{i<j} G\left(\vec{u}_{i}, \vec{u}_{j}\right)+\sum_{i<j} G\left(\vec{v}_{i}, \vec{v}_{j}\right)-\sum_{i j} G\left(\vec{u}_{i}, \vec{v}_{j}\right)\right.}\right) .
$$

Here, $Z_{2 n}$ is a microcanonical partition function of a classical two-component neutral Coloumb gas of $2 n$ particles, and $G(\vec{x}, \vec{y})$ is an interaction potential, which is proportional to Green's function of the Laplace operator on $\Omega$. The most familiar case is when $\Omega=[-\infty, \infty]_{x} \times$ $[-\infty, \infty]_{\tau}$ and $G(\vec{x}, \vec{y})=\ln |\vec{x}-\vec{y}|$.

To evaluate $Z(g)$ nonperturbatively in $g$, we introduce an auxiliary function $W(\alpha)$, which should be understood as a certain distribution function, and is defined in such a way that its $n$th moment equals $Z_{2 n}$.

$$
Z_{2 n}=\int W(\alpha) \alpha^{n} d \alpha
$$

One can use the Hankel transformation [19] to compute $Z(g)$ from $W(\alpha)$ as

$$
Z(g)=\int_{0}^{\infty} W(\alpha) I_{0}(2 g \sqrt{\alpha}) d \alpha
$$

This equation equation can be verified using the Taylor expansion of the modified Bessel function $I_{0}(x)$ and Eq. (3). Formulation of the auxiliary "problem of moments" allows one to avoid calculating $Z(g)$ order by order, and can be viewed as a tool to sum the perturbation series in Eq. (2) to all orders.

Function $G(\vec{x}, \vec{y})$ is real and symmetric, so it can be diagonalized on $\omega$ by solving the eigenvalue equations

$$
\int_{\omega} G(\vec{x}, \vec{y}) \Psi_{f}(\vec{y}) d^{2} \vec{y}=G(f) \Psi_{f}(\vec{x})
$$

Here $f$ is an integer index, which goes from 1 to $\infty . \Psi_{f}(\vec{x})$ can be chosen to be real and normalized according to $\int_{\omega} \Psi_{f}(\vec{x}) \Psi_{k}(\vec{x}) d^{2} \vec{x}=\delta(f, k)$. Then, $G(\vec{x}, \vec{y})$ is given by $G(\vec{x}, \vec{y})=\sum_{f=1}^{f=\infty} G(f) \Psi_{f}(\vec{x}) \Psi_{f}(\vec{y})$. Such decomposition is similar to the diagonalization of a symmetric matrix using its eigenvectors and eigenvalues. We have

$$
\begin{gathered}
Z_{2 n}=\int_{\omega} \ldots \int_{\omega} d^{2} \vec{u}_{1} \ldots d^{2} \vec{v}_{n} e^{\sum_{f} \frac{G(f)}{2 K}\left[\left(\sum_{i=1}^{i=n} \Psi_{f}\left(\vec{u}_{i}\right)-\Psi_{f}\left(\vec{v}_{i}\right)\right)^{2}-\sum_{i=1}^{i=n}\left(\Psi_{f}\left(\vec{u}_{i}\right)^{2}+\Psi_{f}\left(\vec{v}_{i}\right)^{2}\right)\right]}= \\
\int_{\omega} \ldots \int_{\omega} d^{2} \vec{u}_{1} \ldots d^{2} \vec{v}_{n} \prod_{f=1}^{f=\infty} \frac{\int_{-\infty}^{\infty} d t_{f} e^{-\frac{t_{f}^{2}}{2}} e^{\sum_{i} t_{f} \sqrt{\frac{G(f)}{K}}\left(\Psi_{f}\left(\vec{u}_{i}\right)-\Psi_{f}\left(\vec{v}_{i}\right)\right)-\frac{G(f)}{2 K}\left(\Psi_{f}\left(\vec{u}_{i}\right)^{2}+\Psi_{f}\left(\vec{v}_{i}\right)^{2}\right)}}{\sqrt{2 \pi}} .
\end{gathered}
$$

To go from the first to the second line in Eq. (6) we introduced the Hubbard-Stratonovich variables $t_{f}$. Integration over $d^{2} \vec{u}_{1} \ldots d^{2} \vec{v}_{n}$, is now straightforward since all $\vec{u}-$ and $\vec{v}-$ integrals are identical.

$$
Z_{2 n}=\left(\prod_{f=1}^{f=\infty} \frac{\int_{-\infty}^{\infty} e^{-\frac{t_{f}^{2}}{2}} d t_{f}}{\sqrt{2 \pi}}\right) g\left(\left\{t_{f}\right\}\right)^{n} g\left(\left\{-t_{f}\right\}\right)^{n}
$$


where

$$
g\left(\left\{t_{f}\right\}\right)=\int_{\omega} d \vec{x} e^{\sum_{f} t_{f} \sqrt{\frac{G(f)}{K}} \Psi_{f}(\vec{x})-\frac{G(f)}{2 K} \Psi_{f}(\vec{x})^{2}} .
$$

From a comparison of Eqs. (3) and (7) we obtain

$$
W(\alpha)=\prod_{f=1}^{f=\infty} \frac{\int_{-\infty}^{\infty} e^{-\frac{t_{f}^{2}}{2}} d t_{f}}{\sqrt{2 \pi}} \delta\left[\alpha-g\left(\left\{t_{f}\right\}\right) g\left(\left\{-t_{f}\right\}\right)\right] .
$$

Equations (8) and (9) have a simple physical interpretation. Consider $\Psi_{f}(\vec{x})$ to be the eigenmodes of the surface vibrations, $t_{f}$ the fluctuating mode amplitudes, and $|G(f)|$ the noise power. Infinite dimensional integral over $\left\{t_{f}\right\}$ variables can be understood as an averaging over fluctuations of the surface. For a particular realization of noise variables $\left\{t_{f}\right\}$, a complex valued surface coordinate at point $\vec{x}$ is given by $h\left(\vec{x} ;\left\{t_{f}\right\}\right)=$ $\sum_{f} t_{f} \Psi_{f}(\vec{x}) \sqrt{G(f) / K}-G(f) \Psi_{f}(\vec{x})^{2} / 2 K$. For each realization of a random surface $\left\{t_{f}\right\}, g\left(\left\{t_{f}\right\}\right)$ is obtained as an integral (8), which can also be written as $g\left(\left\{t_{f}\right\}\right)=$ $\int_{\omega} d^{2} \vec{x} e^{h\left(\vec{x} ;\left\{t_{f}\right\}\right)}$. Hence Eq. (9) can be interpreted as the mapping between the partition function of the SG model (11) and the statistics of random surfaces subject to classical noise. This mapping is the central result of this paper.

In general, $g\left(\left\{t_{f}\right\}\right)$ is a complex number, thus in general, $\alpha$ is defined on a complex plane. Simplifications occur: if all eigenvalues $G(f)$ are negative, then $g\left(\left\{-t_{f}\right\}\right)=$ $g\left(\left\{t_{f}\right\}\right)^{*}, g\left(\left\{t_{f}\right\}\right) g\left(\left\{-t_{f}\right\}\right)=\left|g\left(\left\{t_{f}\right\}\right)\right|^{2}$, and $\alpha$ is always real and positive. Function $W(\alpha)$ can be computed efficiently using Eq. (9) and Monte Carlo simulations. In the first step one solves integral equations (5) numerically to obtain eigenfunctions $\Psi_{f}(\vec{x})$ and eigenvectors $G(f)$. Then one samples random numbers $\left\{t_{f}\right\}$ from the Gaussian ensemble, and plots the histogram of the results for $g\left(\left\{t_{f}\right\}\right) g\left(\left\{-t_{f}\right\}\right)$. Each point on a histogram requires a computation of only two integrals, and $W(\alpha)$ can be evaluated to arbitrary precision.

Note, that this simple numerical evaluation of $W(\alpha)$ allows one to extract $Z(g)$ for all values of $g$ using Eq. (44). While conventional large-scale Monte Carlo simulations [20, 21] can be used to extract properties of SG models, they require separate simulation for each value of $g$. Application of such methods to the calculation of DFIFA described below would also require analytic continuation of numerical results to imaginary values of $g$ [15], which is numerically unstable. In addition, the mapping in Eqs. (8) and (91) does not only simplify numerical simulations, but can also be used to obtain analytical results (see below).

\section{APPLICATIONS TO INTERFERENCE OF LOW-DIMENSIONAL GASES}

We now apply a general formalism developed to a particular problem of the interference of low-dimensional

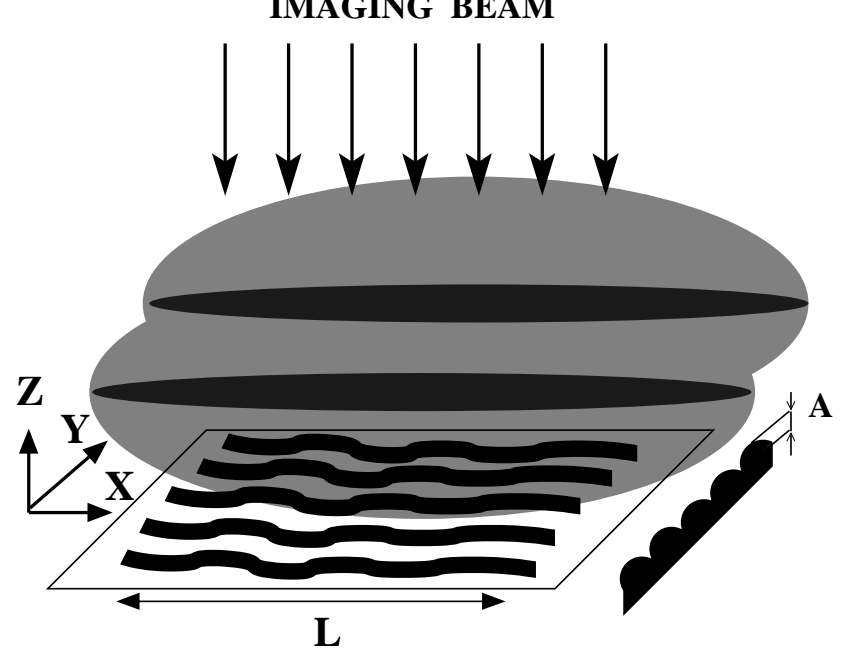

FIG. 1: Simplified setup of interference experiments with 1D Bose liquids (see, e.g., Refs. [1], 12]). Two parallel condensates are extended in the $x$ direction. After atoms are released from the trap, clouds are imaged by the laser beam propagating along the $z$ axis. Meandering structure of the interference pattern arises from phase fluctuations along the condensates. The net interference amplitude $A$ is defined from the density integrated along the section of length $L$. For the analogous 2D setup, see, e.g., Fig. 2 of Ref. [14].

Bose gases 11, 12, 13, 14, 15, 16. Typical experimental setup for interference of low-dimensional gases with open boundary conditions (OBC) is shown in Fig. 1. Two parallel condensates are extended in the $x$ direction. After atoms are released from the trap, clouds expand predominantly in the transverse direction. After sufficient time of flight clouds overlap, and the laser beam propagating along the $z$ axis takes an absorption image. Fluctuations of the relative phase result in fluctuations of the minima positions for different $x$. For each $y$, the image can be integrated along the $x$ direction to obtain the integrated fringe amplitude $A$. One experimental image can be used to extract information for different values of $L$. Many images are still required to obtain distribution functions for each $L$.

For two identical 1D clouds higher moments of the fringe amplitude $A$ can be written as [14]

$$
\left\langle|A|^{2 n}\right\rangle=A_{0}^{2 n} Z_{2 n}, \text { where } A_{0}=\sqrt{C \rho^{2} \xi_{h}^{1 / K} L^{2-1 / K}} .
$$

Here $\rho$ is the density, $L$ is the imaging length, $\xi_{h}$ is the healing length, and $C$ is a constant of the order of unity. For OBC $Z_{2 n}$ is given [14] by Eq. (2) with $\Omega=[-\infty, \infty]_{x} \times[0, \beta]_{\tau}$ (and periodic boundary conditions in $\tau$ ) and $\omega=[0,1]_{x}$ (and fixed $\tau$ ). Equation (10) has been derived neglecting the shot noise, which arises due to a finite number of particles in the interfering clouds [16, 22]. In what follows, we will be interested in the distribution functions $W(\alpha)$ of a positive variable $\alpha=|A|^{2} / A_{0}^{2}$ defined by Eq. (31), or of its normalized version $\tilde{\alpha}=|A|^{2} /\left\langle A^{2}\right\rangle$, defined by 


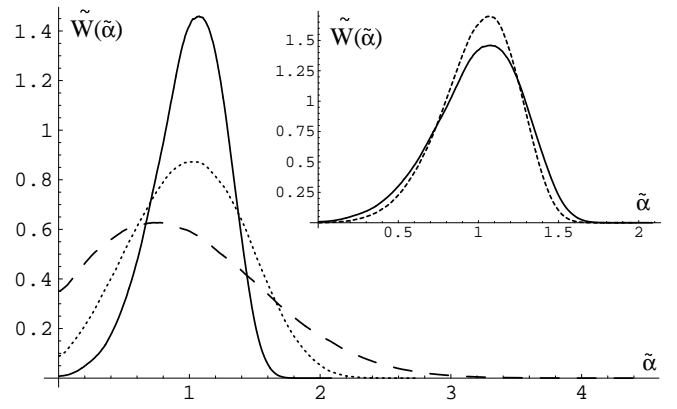

FIG. 2: Distribution functions of the normalized interference amplitude $\tilde{W}(\tilde{\alpha})$ at $T=0$ for $1 \mathrm{D}$ gases with open boundary conditions, shown for Luttinger parameters $K=$ 2 (dashed), $K=3$ (dotted), and $K=5$ (solid). The inset shows a comparison between open (solid) and periodic (dashed) boundary conditions for $K=5$.

$Z_{2 n} / Z_{2}^{n}=\int_{0}^{\infty} \tilde{W}(\tilde{\alpha}) \tilde{\alpha}^{n} d \tilde{\alpha}$. For zero temperature $Z_{2 n}$ depends only on the Luttinger parameter $K$, which describes [23, 24] the long-distance behavior of boson correlation functions, given by $\left\langle a^{\dagger}(x) a(0)\right\rangle \sim \rho\left(\xi_{h} / x\right)^{1 / 2 K}$. For bosons $K$ ranges from $K=1$ (strong interactions) to $K=\infty$ (weak interactions). For OBC and zero temperature $G(\vec{x}, \vec{y})$ equals $G(\vec{x}, \vec{y})=\log |\vec{x}-\vec{y}|$, while for PBC considered in Ref. [15], $G^{\text {per }}(\vec{x}, \vec{y})=\ln \frac{1}{\pi} \sin \pi|\vec{x}-\vec{y}|$. While $A_{0}$ depends on $L$, for zero temperature distribution $\tilde{W}(\tilde{\alpha})$ does not depend on $L$, but depends only on $K$. For nonzero temperature, $Z_{2 n}$ depends on $K$ and the thermal length $\xi_{T}=\hbar v_{s} /\left(k_{B} T\right)$, where $v_{s}$ is the sound velocity: $G\left(\vec{x}, \vec{y}, \xi_{T} / L\right)=\ln \left(\frac{\xi_{T}}{\pi L} \sinh \frac{\pi|\vec{x}-\vec{y}| L}{\xi_{T}}\right)$.

For $2 \mathrm{D}$, one can use a similar approach to describe the contrast distribution at finite temperature below the BKT transition. In this case, correlation functions are given by $\left\langle a^{\dagger}(r) a(0)\right\rangle \sim \rho\left(\xi_{h} / r\right)^{\eta(T)}$, where $\eta(T)=$ $m T /\left(2 \pi \hbar^{2} \rho_{s}(T)\right)$ depends on the temperature and the superfluid density $\rho_{s}(T)$. The BKT transition happens at the universal value $\eta_{c}\left(T_{c}\right)=1 / 4$. To keep a connection to the $1 \mathrm{D}$ case, we will use $K=1 /(2 \eta(T))$, and restrict our attention to $K>K_{c}=2$. For $2 \mathrm{D}$ with the aspect ratio of the imaging area equal to unity, $\vec{u}_{i}$ and $\vec{v}_{i}$ in Eq. (2) are defined on a square $\omega=[0,1]_{x} \times[0,1]_{\tau}$ with $G(\vec{x}, \vec{y})=\ln |\vec{x}-\vec{y}|$.

In the limit $K \rightarrow \infty$, one can expand the exponent of Eq. (8) in the Taylor series. Then $\alpha=g\left(\left\{t_{f}\right\}\right) g\left(\left\{-t_{f}\right\}\right)$ is linearly related to roughness, or mean square fluctuation of the surface, as defined in Ref. 25]. For PBC the noise has a $1 / f$ power spectrum, since $G^{\text {per }}(x, y)=$ $\ln \frac{1}{\pi} \sin \pi|x-y|=-\ln 2 \pi-\sum_{f=1}^{f=\infty}(\cos 2 \pi f x \cos 2 \pi f y+$ $\sin 2 \pi f x \sin 2 \pi f y) / f$. This results in the Gumbel statistics of the roughness [15, 16, 25, 27]: $\tilde{W}_{G}(\tilde{\alpha})=$ $K \exp \left(x-e^{x}\right)$, where $x=K(\tilde{\alpha}-1)-\gamma$ and $\gamma=0.577$ is the Euler constant. This provides the analytical proof of the conjecture made in Ref. [15], that the distribution function in this case is given by one of the extreme value statistical distributions, the Gumbel function [26].

In what follows we perform simulations of $W(\alpha)$ with

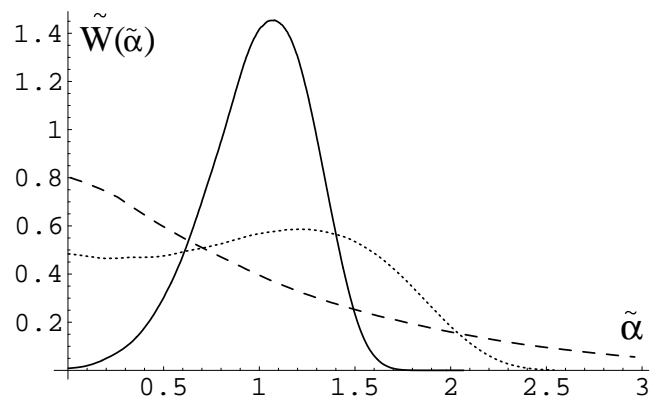

FIG. 3: Distribution functions of the normalized interference amplitude $\tilde{W}(\tilde{\alpha})$ for a 1D Bose gas with open boundary conditions at nonzero temperature and $K=5$. Different curves correspond to ratios $K \xi_{T} / L=\infty$ (solid), $K \xi_{T} / L=1$ (dotted), and $K \xi_{T} / L=0.25$ (dashed). $\xi_{T}$ is the thermal correlation length, $K$ is the Luttinger parameter, and $L$ is the imaging length.

up to $N=10^{6}-10^{7}$ realizations of $\left\{t_{f}\right\}$ and smoothen the data. We use a finite value of $f_{\max }$ and check for convergence with $f_{\max }$, typically $\sim 30$. $\langle\alpha\rangle$ is always kept within $1 \%$ from its expected value. For most of the presented results, all eigenvalues $G(f)$ are negative, and Eq. (9) can be directly applied. Special care should be taken of the $1 \mathrm{D}$ case with nonzero temperatures, since one of the eigenvalues can be positive. This situation can be handled by subtracting a sufficiently large positive constant $C$ from $G\left(\vec{x}, \vec{y}, \xi_{T} / L\right)$, which makes all eigenvalues negative. According to Eqs. (2) and (3), this leads to rescaling of $\alpha$ by a factor $e^{-C / K}$, which can be easily taken into account.

In Fig. 2 we show distribution functions of the normalized interference amplitude $\tilde{W}(\tilde{\alpha})$ at $T=0$ for $1 \mathrm{D}$ gases with $\mathrm{OBC}$ for various $K$. The inset shows a comparison between $\mathrm{OBC}$ and $\mathrm{PBC}$ for $K=5$. In Fig. 3, we show distribution functions of the normalized interference amplitude for a $1 \mathrm{D}$ gas with $\mathrm{OBC}$ at nonzero temperature and $K=5$. For $\xi_{T} K / L \ll 1$ distribution is Poissonian [14, 15, 16] and wide, while for $K \gg 1$ and $\xi_{T} K / L \gg 1$ it is very narrow. Evolution of the full distribution function of the visibilities as $L$ is varied can be used to precisely measure the thermal length $\xi_{T}$, and to extract the temperature. As seen in Fig. 3, at $T \neq 0$ the distribution function has characteristic features, i.e., it is generally nonsymmetric and can have a minimum. These features can be used to distinguish the intrinsic noise due to fluctuations of the phase from technical noise. Finally, in Fig. 4 we show distribution functions of the normalized interference amplitude for a $2 \mathrm{D}$ gas with an aspect ratio of imaging area equal to unity and $\mathrm{OBC}$ below the BKT temperature. Above the BKT temperature, distribution functions become Poissonian for $L \gg \xi$, where $\xi$ is the correlation length. In $2 \mathrm{D}$ one cannot describe the crossover at $L \sim \xi$ similar to $1 \mathrm{D}$, since the action which describes the fluctuations of the phase is not quadratic in this region, and Eq. (2) does not hold. 


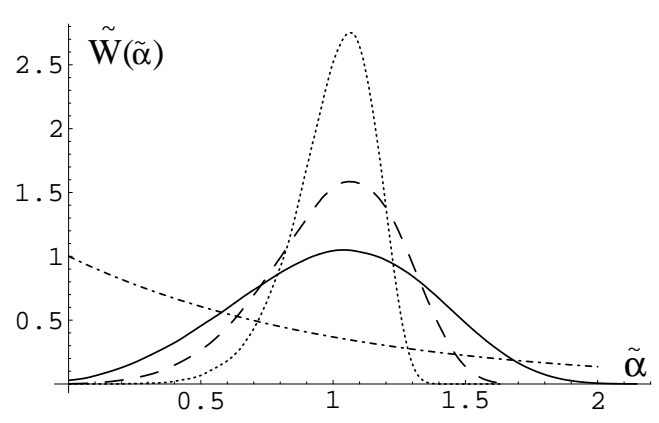

FIG. 4: Distribution functions of the normalized interference amplitude $\tilde{W}(\tilde{\alpha})$ for a two-dimensional Bose gas with the aspect ratio of the imaging area equal to unity and open boundary conditions. Temperature is below the BerezinskiiKosterlitz-Thouless (BKT) transition temperature. Different curves correspond to $\eta(T)=\eta_{c}\left(T_{c}\right)=1 / 4$ (the BKT transition point, solid), $\eta(T)=1 / 6$ (dashed line), and $\eta(T)=1 / 10$ (dotted line). Above the BKT transition temperature the distribution function is Poissonian (dot-dashed line).

\section{CONCLUSIONS}

To summarize, we introduced a class of sine-Gordon models, for which an interaction term is present in a spa- tial region different from the domain over which the noninteracting part is defined. We developed a general mapping of such sine-Gordon models and related Coulomb gases to statistical properties of random surfaces, which can be used to calculate their partition functions nonperturbatively. As a specific application of our approach, we considered interference experiments with two independent low-dimensional Bose gases. We calculated full distribution functions of the interference amplitude for $1 \mathrm{D}$ and 2D gases with open boundary conditions and nonzero temperatures. Full distribution functions of interference fringe visibilities can be used for thermometry.

We thank M. Mueller and A. Polkovnikov for useful discussions. This work was partially supported by NSF Grant No. DMR-0705472, MIT-Harvard CUA, and AFOSR. V.G. is also partially supported by Swiss National Science Foundation, Grant No. PBFR2-110423.
[1] V. L. Berezinskii, Sov. Phys. JETP 32, 493 (1971);34, 610 (1972).

[2] J. M. Kosterlitz and D. J. Thouless, J. Phys. C 6, 1181 (1973).

[3] A. O. Gogolin, A. A. Nersesyan and A. M. Tsvelik, Bosonization and Strongly Correlated Systems (Cambridge University Press, Cambridge, England, 1998).

[4] G. Refael, E. Demler, Y. Oreg, and D. S. Fisher, Phys. Rev. B 75, 014522 (2007).

[5] P. Fendley, H. Saleur, and N. P. Warner, Nucl. Phys. B 430, 577 (1994).

[6] S. Chakravarty, Phys. Rev. Lett. 49, 681 (1982); A. Schmid, Phys. Rev. Lett. 51, 1506 (1983).

[7] C. L. Kane and M. P. A. Fisher, Phys. Rev. B 46, 15233 (1992).

[8] L. A. Takhtadjan and L. D. Faddeev, Sov. Theor. Math. Phys. 25, 147 (1975)

[9] F. J. Wegner and A. Houghton, Phys. Rev. A 8, 401 (1973).

[10] R. Klesse and A. Stern, Phys. Rev. B 62, 16912 - 16925 (2000)

[11] T. Schumm et al., Nature Physics 1, 57 (2005).

[12] S. Hofferberth et al., Nature Physics 2, 710 (2006).

[13] Z. Hadzibabic et al., Nature 441, 1118 (2006).

[14] A. Polkovnikov, E. Altman and E. Demler, Proc. Natl. Acad. Sci. USA 103, 6125 (2006).

[15] V. Gritsev, E. Altman, and E. Demler and A.
Polkovnikov, Nature Physics 2, 705 (2006).

[16] A. Imambekov, V. Gritsev, and E. Demler, in Proceedings of the 2006 Enrico Fermi Summer School on Ultracold Fermi Gases, Varenna, Italy, June 2006, organized by M. Inguscio, W. Ketterle and C. Salomon; arXiv:cond-mat/0703766 v1.

[17] V. V. Bazhanov, S. L. Lukyanov, and A. B. Zamolodchikov, Commun. Math. Phys. 177, 381 (1996); 190, 247 (1997); 200, 297 (1999).

[18] S. T. Chui and P. A. Lee, Phys. Rev. Lett. 35, 315 (1975).

[19] A. D. Polyanin and A. V. Manzhirov, Handbook of Integral Equations (CRC Press, Boca Raton, 1998).

[20] M. Hasenbusch, M. Marcu, and K. Pinn, Physica A 211, 255 (1994).

[21] A. Sanchez, A. R. Bishop, and E. Moro, Phys. Rev. E 62, 3219 (2000).

[22] A. Polkovnikov, Europhys. Lett. 78, 10006 (2007).

[23] F. D. M. Haldane, Phys. Rev. Lett. 47, 1840 (1981).

[24] M. A. Cazalilla, J. Phys. B 37, S1 (2004).

[25] T. Antal, M. Droz, G. Gyorgyi, and Z. Racz, Phys. Rev. Lett. 87, 240601 (2001).

[26] E. J. Gumbel, Statistics of the Extremes (Columbia University Press, New York, 1958).

[27] E. Bertin, Phys. Rev. Lett. 95, 170601 (2005); E. Bertin and M. Clusel, J. Phys. A 39, 7607 (2006). 